\title{
Simulación y análisis de desempeño de protocolos unicast para Redes VANET
}

\section{Simulation and Performance Analysis of Protocols for Unicast Vanet}

\section{Gonzalo Jiménez Pinto}

Ingeniero Electrónico, estudiante de la Maestría en Ciencias de la Información y las Comunicaciones de la Universidad Distrital Francisco José de Caldas. Bogotá, Colombia.

gjimenezp@udistrital.edu.co

\section{Danilo López}

Ingeniero electrónico, magíster en Teleinformática. Docente e investigador de la Universidad Distrital Francisco José de Caldas. Bogotá, Colombia. dalopezs@udistrital.edu.co

\section{Luis F. Pedraza}

Ingeniero Electrónico, magíster en Ciencias de la Información y las Comunicaciones. Docente e investigador de la Universidad Distrital Francisco José de Caldas. Bogotá, Colombia.

lfpedrazam@udistrital.edu.co

Clasificación del artículo: Investigación (conciencias)

Fecha de recepción: 4 de agosto de 2011

Fecha de aceptación: 28 de noviembre de 2011

Palabras clave: AODV, DSDV, DSR, rendimiento, red vehicular Ad-Hoc.

Keywords: AODV, DSDV, DSR, performance, vehicular Ad-Hoc networks.

\section{RESUMEN}

Los cambios constantes de la topología en las redes VANET (Vehicular Ad-Hoc Networks), la velocidad de movimiento de los nodos, entre otros problemas, generan desafíos tecnológicos en el enrutamiento de estas redes, lo que ha llevado a la implementación de diferentes protocolos. El estudio y análisis de las características y desempeño de los protocolos comúnmente empleados por las VANETs, permite la formulación de posibles soluciones a esos desafíos. Para hacer esto se precisa del uso de herramientas, como los simuladores de redes. En este trabajo se presenta una definición de las VANETs, así como de sus características, una clasificación y descripción de algunos protocolos comúnmente usados por estas y se realiza una investigación mediante la simulación del comportamiento de una red, para finalmente, presentar el análisis de resultados y conclusiones del comportamiento de los protocolos AODV, DSR y DSDV, se escogen estos por ser los más usados por su rendimiento. 


\section{con-ciencias}

\section{ABSTRACT}

The constant changes in network topology VANET (Vehicular Ad-Hoc Networks), the moving speed of nodes, among other problems, generate technological challenges in the routing of these networks, which has led to the implementation of different protocols. The study and analysis of the characteristics and performance of the protocols commonly used by VANETs, allows the formulation of possible solutions to these challenges. To do this requires the use of tools such as simulators for networks. This paper presents a definition of VANETs and their characteristics, classification and description of some commonly used protocols for this and an investigation is conducted by simulating the behavior of a network, to finally present the analysis of results and conclusions the behavior of protocols AODV, DSR and DSDV are chosen these as the most commonly used for performance.

\section{INTRODUCCIÓN}

Un aspecto atractivo de la tecnología para la industria se presenta en los sistemas de comunicación entre vehículos. Una de las principales aplicaciones en estudio y desarrollo es, las redes de comunicaciones vehiculares [1]. Con la adopción del estándar 802.11a [2], se plantea una de las aplicaciones de las redes (Mobile Ad-hoc Networks) MANETs, las (Vehicular Ad-Hoc Networks) VANETs, que son redes móviles que suministran información sobre el tráfico vehicular y pueden ser usadas, entre otras cosas, para evitar congestiones y mejorar la seguridad de los pasajeros [3]. Las estadísticas muestran que en el mundo, los accidentes de tráfico ocupan el noveno lugar en el índice de mortalidad [4]. Una de las características de estas redes es que no se cuenta con un nodo central, ya que cualquiera de los nodos, en determinado momento, debe actuar como una estación o nodo central o como un router. Por otra parte, el movimiento arbitrario de los nodos hace que se produzca un cambio constante en la topología de la red, lo que afecta las condiciones de la comunicación. Todo esto propone retos tecnológicos dentro de los que se identifica la limitación en el ancho de banda [5], convirtiéndose en tema de constante investigación el manejo y asignación del ancho de banda. Por otra parte, están, el tiempo de respuesta de los conductores y la velocidad de diseminación de los mensajes, así como la recepción de señal por nodos adyacentes [6]. Debido a esto se busca algoritmos para el control de la diseminación de los mensajes, que serian manejados por la policía y organismos de emergencia. También hay que tener en cuenta la redundancia en las comunicaciones [7], ya que esto congestiona la red. Todo esto ha llevado a la formulación de diferentes protocolos. El estudio y análisis del rendimiento de estos ha permitido proponer soluciones y mejoras. Para ello se recurre a herramientas para la simulación de redes, una de ellas es, ns-2 [8]. En este trabajo se simula una red, tomando como referencia un escenario, basado en la información de tráfico vehicular en la ciudad de Bogotá, buscando un escenario que se acerque a la realidad. Los protocolos que se van a analizar son: Ad-hoc On-Demand Distance Vector (AODV) [9], Dynamic Source Routing (DSR) [10] y Destination-Sequenced Distance Vector (DSDV) [11]. Se escogen estos protocolos por ser algunos de los más representativos y que muestran mejores características [12]. Finalmente, se presentará una comparación del rendimiento de estos protocolos.

\section{VANETS: CARACTERÍSTICAS}

Las VANETs son redes en las que sus nodos son vehículos, la principal característica de estas redes es el cambio constante en su topología. Este cambio se produce por la movilidad de los vehículos. Uno de los inconvenientes está en la velocidad de movimiento de los nodos [13]. Vehículos en la misma dirección normalmente tienen la misma velocidad por lo que la comunicación entre ellos puede durar más tiempo, no así cuando van en direcciones opuestas. Por otra parte, las velocidades están sujetas a las normas de transito, igual que las 
rutas, en la zona o escenario. Esto hace que tanto la topología de red, como algunos de los parámetros de movilidad sean predecibles.

En las redes vehiculares se da gran importancia a la fiabilidad de la información así como su obtención en tiempo real. En el caso de los organismos de emergencia esta información puede ayudarlos a llegar a tiempo a los lugares de siniestros y para los usuarios en general presenta la posibilidad de evitar situaciones, como congestiones o accidentes vehiculares.

Dentro de las aplicaciones de las VANETs está, entre otras, el acceso a Internet por parte de los pasajeros, servicios de video juegos y control del tráfico vehicular en tiempo real.

Al igual que otros ambientes de comunicación, las VANETs, están regidas por un estándar, que en este caso es el 802.11, y específicamente el 802.11p.

\section{ESTÁNDAR 802.11}

El estándar 802.11 es la norma creada para reglamentar las comunicaciones inalámbricas. El grupo de trabajo de IEEE 802.11 trabaja en el 802.11p [14], que será la base para las comunicaciones dedicadas de corto alcance. Dedicated Short Range Communications (DSRC), en los Estados Unidos. Por otra parte en Europa se viene desarrollando el proyecto CIVIS (Cooperative Vehicle Infrastructure Systems) [15] donde 60 compañías se han unido para crear un sistema inteligente de transporte. La norma $802.11 \mathrm{p}$ proporciona dispositivos inalámbricos capaces de realizar intercambios de corta duración para establecer comunicaciones entre vehículos y/o con una estación de tierra, a altas velocidades. Uno de los objetivos principales de esta norma es minimizar la latencia, por lo que se han hecho pruebas que muestran que este protocolo puede ofrecer latencias inferiores a las de las redes celulares, algo que es de suma importancia a la hora de requerir respuestas rápidas. Actualmente el ancho de banda medio, requerido para las aplicaciones que utilizaran este protocolo, DSRC y CIVIS es de 6 Mbps y una cobertura de $300 \mathrm{~m}$ pero se espera que se llegue a $27 \mathrm{Mbps}$ y una cobertura de $1 \mathrm{Km}[16]$.

\section{CLASIFICACIÓN DE PROTOCOLOS Y SUS CARACTERÍSTICAS}

Debido a las características propias de las VANETs, los protocolos usados en ellas deben tener en cuenta dos aspectos importantes, uno es el alcance, es decir, a cuantos nodos se envían mensajes o paquetes de información, y otro, es la manera como se llegará al destino, en otras palabras, el descubrimiento de la ruta. Basándose en el alcance los protocolos se dividen en multicast y unicast. Los protocolos multicast envían información a múltiples destinos, mientras que en los unicast la envían a un único destino. Este trabajo se centra en los protocolos unicast.

Hay diferentes maneras de clasificar los protocolos, dependiendo de sus características.

Jerárquico vs plano: en esta clasificación se tiene en cuenta el nivel al que pertenecen los nodos. En los jerárquicos, los nodos están en diferentes niveles y su rol depende del nivel en que se encuentren, mientras que en el plano todos los nodos tienen asignados los mismos roles, por estar en un solo nivel [17]. Un ejemplo de protocolos clasificados como planos son los protocolos AODV y DSR, por otra parte un protocolo jerárquico es el protocolo Cluster Gateway Switch Routing Protocol (CGSR) [11].

Geográficos vs No geográficos: esta clasificación depende de si se tiene en cuenta la posición geográfica de los nodos o no.

Reactivos vs proactivos: esta clasificación está basada en las características del establecimiento de ruta. Los reactivos son los que crean rutas por demanda, es decir, solo cuando es necesario [18]. Por el contrario, en los protocolos proactivos se están buscando rutas continuamente, así no se necesiten [18]. También hay protocolos híbridos, que combinan características reactivas y proactivas.

Dentro de los protocolos reactivos están:

AODV: el protocolo AODV propuesto en 1999 [9], es un protocolo para redes ad-hoc, que establece la ruta por demanda, es decir, que la ruta no está preestablecida. Pero esto no quiere decir que los 


\section{con-ciencias}

mensajes sean enviados a ciegas, ya que el nodo receptor válida las rutas comparando el número de secuencia. Si un nodo quiere establecer comunicación con otro nodo, y la ruta no está establecida, envía un mensaje broadcast de solicitud de ruta ROUTE REQUEST (RREQ) [9]. Los nodos por los que tiene que pasar este mensaje registran la información sobre la ruta inversa hacia el nodo de inicio. A cada nodo se asocia una tabla de encaminamiento que contiene el identificador de origen, el identificador de destino, el número de secuencia de origen, el número de secuencia de destino, el identificador de emisión, el tiempo de vida y el contador de salto. Al llegar a un nodo y encontrar la ruta válida se comprueba el número de secuencia (DestSeqNum), si este número es mayor al número de secuencia guardado, se considera que la ruta es válida, y este nodo envía un mensaje tipo ROUTE REPLY (RREP), con la información sobre la ruta inversa hacia el nodo de inicio [11]. Una vez establecida la ruta esta se mantiene por un tiempo, para no tener que realizar todo el proceso cada vez que se envía un paquete. Si un nodo intermedio se mueve, la topología cambia, por lo que este nodo ya no será utilizado y el nodo inmediatamente anterior, de fuente a destino, notifica, mediante un mensaje de error en la ruta, Route Error (RERR), el movimiento del nodo y así el nodo de inicio deberá volver a comenzar el descubrimiento de ruta. También podría darse el caso del movimiento del nodo de inicio, en este caso el mismo nodo debe reiniciar el descubrimiento de ruta. Por otra parte debido a que muchos nodos están transmitiendo se pueden recibir números de secuencia de destino repetidos, en ese caso los números repetidos se eliminan. Este protocolo tiene como ventaja, que puede ser utilizado en redes altamente escalables. Una desventaja es el incremento del número de secuencia y el decremento de número de saltos, de una manera engañosa, que puede generar errores en la ruta [19].

DSR: el protocolo DSR [10] es un protocolo diseñado específicamente para redes inalámbricas multi-hop y redes ad-hoc. Este protocolo, propuesto en 1996 [10], tiene dos componentes básicos que son: el descubrimiento de ruta, que es el mecanismo mediante el cual, si un nodo desea enviar un mensaje a un nodo desconocido, se obtiene una ruta de destino. El otro componente es el mantenimiento de ruta, que es el mecanismo mediante el cual, si un nodo quiere enviar un mensaje a un nodo destino, detecta cuando la topología ha cambiado, por el movimiento de los nodos, y busca otra ruta o llama al descubrimiento de ruta para establecer una nueva [10], [20]. Este es un protocolo que funciona básicamente como el AODV pero a diferencia de él, no genera una tabla de enrutamiento inversa. Este protocolo se basa en el encaminamiento desde el origen. Para esto se inserta una cabecera con la información sobre los nodos que se deben recorrer para llegar al destino. Una de las características de este protocolo es que con las optimizaciones disponibles puede ser utilizado en redes pequeñas [21], con la ventaja de un menor overhead, respecto a AODV. TORA: este es un protocolo propuesto en 1996, para mejorar el rendimiento de las redes altamente dinámicas. Funciona básicamente como DSR, pero a diferencia de él, elimina las rutas inválidas, busca una nueva ruta y la establece, en un solo paso, mientras que DSR lo hace en tres pasos [22].

Dentro de los protocolos proactivos están:

DSDV: el protocolo DSDV, propuesto en 1994 [11], está basado en el algoritmo de distribución de Bellman-Ford (BDF), que comparado con el método de actualización del estado de enlace es computacionalmente más eficiente y requiere mucho menos espacio de almacenamiento [22]. En este protocolo cada entrada contiene un número de secuencia. Este número es emitido por el destino y el emisor tiene que enviar la próxima actualización de este número. La información de ruta es distribuida en paquetes completos con periodicidades cortas y las actualizaciones incrementales se envían con mayor periodicidad [11], [22].

FSR: el protocolo FSR es un protocolo basado en una técnica de alcance multinivel, que emplea una tabla de enrutamiento. Mediante esta técnica se reduce el overhead cuando hay cambios rápidos en la red, como los que se presentan en redes VANET. Constantemente se están emitiendo las actualizaciones de enlace, de acuerdo con una frecuencia especificada por el método de alcance. Las actualizaciones de estado de enlace se propagan con diferentes periodicidades, dependiendo de la distancia a la que se encuentre el nodo vecino. La 
información se trasmite con mayor periodicidad a los nodos más cercanos, por lo que los nodos más alejados, fuera de la red, no tienen la información más actualizada sobre el estado de los enlaces y por tanto esta es imprecisa. Dentro de las ventajas de este protocolo están: sencillez, robustez y cambio parcial de actualización de enrutamiento con nodos vecinos [18].

LAR (Location Aiden Routing): propuesto en
1998 [23], este protocolo usa el mismo método de descubrimiento de ruta que el protocolo DSDV, pero a diferencia de él, la información no se envía a todos los vecinos, sino que basándose en la información geográfica, la inundación de la red se hace de manera controlada.

A continuación se presenta en la Tabla 1, un resumen de algunas de las características de protocolos unicast.

Tabla 1. Resumen de características de algunos protocolos

\begin{tabular}{|l|c|c|c|c|c|c|}
\hline \multicolumn{1}{|c|}{ Protocolo } & AODV & DSR & FSR & TORA & DSDV & LAR \\
\hline Basado en topología & $\mathrm{X}$ & & $\mathrm{X}$ & & $\mathrm{X}$ & $\mathrm{X}$ \\
\hline Monocanal & $\mathrm{X}$ & $\mathrm{X}$ & & $\mathrm{X}$ & $\mathrm{X}$ & $\mathrm{X}$ \\
\hline Proactivo & & & $\mathrm{X}$ & & $\mathrm{X}$ & $\mathrm{X}$ \\
\hline Reactivo & $\mathrm{X}$ & $\mathrm{X}$ & & $\mathrm{X}$ & & \\
\hline Tabla de ruta & & & $\mathrm{X}$ & & & \\
\hline Latencia alta & $\mathrm{X}$ & $\mathrm{X}$ & & & & \\
\hline Número de rutas alto & $\mathrm{X}$ & $\mathrm{X}$ & & & & \\
\hline Independencia de ruta & $\mathrm{X}$ & $\mathrm{X}$ & & & & \\
\hline Calidad de servicio & $\mathrm{X}$ & $\mathrm{X}$ & $\mathrm{X}$ & $\mathrm{X}$ & & \\
\hline Encaminamiento en origen & & $\mathrm{X}$ & & & & \\
\hline Adecuado en redes altamente densas & $\mathrm{X}$ & & $\mathrm{X}$ & $\mathrm{X}$ & & \\
\hline
\end{tabular}

Haciendo una comparación de trabajos anteriores, en [24] se presenta el análisis de los protocolos DSR, DSDV y AODV teniendo en cuenta como parámetros, el número de saltos, cantidad de paquetes perdidos y tiempo de establecimiento de la comunicación. Como resultado se muestra que en comparación con AODV y DSR, DSDV presenta mayor pérdida de paquetes, más retardo, menor número de paquetes entregados y un número mayor de saltos. En [12] se muestra que comparativamente y teniendo en cuenta un escenario de ciudad, AODV y DSR presenta mejores desempeños que FSR, por otra parte, TORA tiene serios problemas de rendimiento, en redes densas. De esto se desprende que los protocolos con mejor rendimiento, son los protocolos AODV y DSR. En [25] se presenta un análisis de protocolos comúnmente usados, como el DSR, con respecto a nuevos protocolos unicast menos conocidos. Como se muestra en la Fig. 1, la incidencia a través del tiempo de los diferentes protocolos, apunta a que una de las posibles soluciones está en los protocolos que tengan en cuenta la posición geográfica.

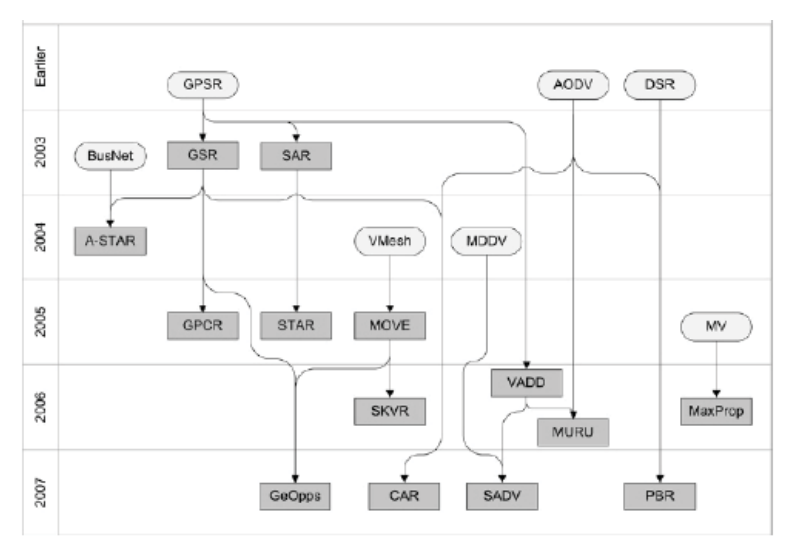

Fig. 1. Protocolos unicast para VANETs y su influencia en el tiempo [25].

\section{METODOLOGÍA}

Para el desarrollo de esta investigación se realizaron simulaciones utilizando la herramienta Network Simulator 2 (ns-2) [8], que es un software que permite simular el comportamiento de las 
redes. Una de las características de este simulador es el manejo de la mayoría de los protocolos comúnmente utilizados, además, los resultados de las simulaciones se pueden obtener de forma gráfica y numérica, lo que permite el análisis de lo observado y es software libre. Algunos de los parámetros por tener en cuenta para la simulación son: número de nodos, velocidad de movimiento de los nodos, tamaño del escenario, tipo de tráfico que se envía, cantidad de paquetes enviados, tiempo que dura el envío de la información y duración de simulación [22].

Para llevar a cabo la simulación de la red, se han elegido los protocolos AODV, DSDV y DSR, por ser algunos de los que presentan mejores características. Utilizando estos protocolos, se escogió como escenario, el tramo de la carrera $7^{\mathrm{a}}$, entre calle 34 a calle 53, de la ciudad de Bogotá. Esto buscando un escenario real.

Por otra parte, se tomaron en cuenta registros de la cantidad de vehículos circulando en sentido sur norte y norte sur, así como de los tiempos promedio requeridos por los mismos para realizar los respectivos recorridos. Los datos sobre el tráfico en la carrera $7^{\mathrm{a}}$ se pueden observar la Tabla 2 que se muestran a continuación.

Tabla 2. Características del tráfico en la Carrera 7 [26].

\begin{tabular}{|c|c|c|}
\hline Indicador & Valor & Unidades \\
\hline Rutas TPC & 98 & $\begin{array}{c}\text { Rutas en el tramo más cargado del cor- } \\
\text { redor }\end{array}$ \\
\hline Buses hora pico & 600 & Bus/hora \\
\hline $\begin{array}{c}\text { Volumen máximo de viajes TPC } \\
\text { (Calle31-Calle 72) }\end{array}$ & 16000 & Pasajeros/hora/sentido \\
\hline Velocidad Promedio TPC & 26 & Vehículos/día/sentido \\
\hline $\begin{array}{c}\text { Volumen máximo diario de vehículos } \\
\text { sentido S-N (Calle 106) }\end{array}$ & 29496 & Vehículos/día/sentido \\
\hline $\begin{array}{c}\text { Volumen máximo diario de vehículos } \\
\text { sentido N-S (Calle 28)1 }\end{array}$ & 22190 & Vehículos \\
\hline $\begin{array}{c}\text { Volumen máximo vehículos sentido } \\
\text { S-N } \\
\text { (Calle 28, Periodo 6:00-9:00 a.m.) }\end{array}$ & 3900 & Vehículos \\
\hline Volumen máximo de vehículos sentido & 7300 & \\
\hline
\end{tabular}

Con estos datos se procedió a programar el software para la simulación, utilizando la herramienta ns-2 [12]. En la Fig. 2 se puede observar una imagen de la simulación en ns- 2 .

Se simula una avenida en dos sentidos, con tres carriles en cada calzada. Cada carril tiene tres metros de ancho.

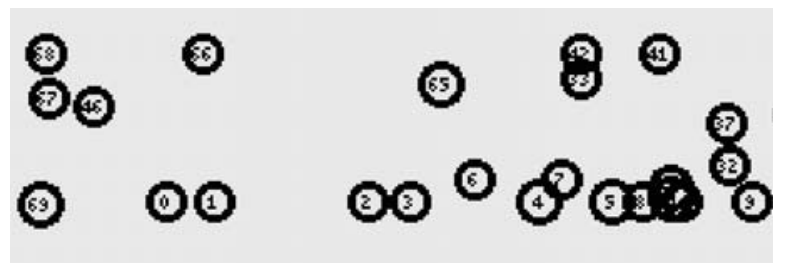

Fig. 2. Imagen de simulación en ns-2.
En la Tabla 3 se muestran los parámetros que se tuvieron en cuenta para la simulación.

Tabla 3. Parámetros de la simulación.

\begin{tabular}{|l|c|}
\hline \multicolumn{1}{|c|}{ Parámetro } & Valor \\
\hline Numero de nodos & 70 \\
\hline Velocidad promedio & $12 \mathrm{a} 60 \mathrm{Km} / \mathrm{h}$ \\
\hline Frecuencia de canal & $2,4 \mathrm{Ghz}$ \\
\hline $\begin{array}{l}\text { Rata de transmisión de } \\
\text { paquetes }\end{array}$ & $2 \mathrm{Mbps}$ \\
\hline Tiempo de simulación & 51 segundos \\
\hline Tamaño del escenario & $1 \mathrm{~km}$ \\
\hline Tipo de antena & Omnidireccional \\
\hline Tipo de protocolo & $\begin{array}{c}\text { AODV, DSR y } \\
\text { DSDV }\end{array}$ \\
\hline
\end{tabular}


Para el análisis se observa el rendimiento de los diferentes protocolos, teniendo en cuenta los cambios en la topología de red. Los parámetros de rendimiento que se tienen en cuenta son:

Troughput: volumen de información que fluye a través de la red.

Tiempo de establecimiento de la comunicación: qué tanto tiempo se demora el establecimiento de la comunicación, usando determinado protocolo.

Cantidad de paquetes perdidos: qué tantos paquetes llegan a su destino.

\section{RESULTADOS}

Se han tomado como referencia, los nodos 1,6 y 63 , buscando observar el comportamiento de los protocolos en nodos intermedios y extremos. El análisis de resultados se fundamenta en la comparación de parámetros observados y graficados en las simulaciones realizadas, nodo a nodo.

Troughput: como se puede ver en las Fig. 3 y 4, en el nodo 1, el troughput es mayor con la aplicación del protocolo AODV, mientras que, con DSR y DSDV no se presenta transferencia alguna. En las Fig. 5 y 6 se observa que el troughput asociado al nodo 6 existe, pero es muy inestable. Para el nodo 63 , con el protocolo AODV se presenta en promedio una mayor transferencia de paquetes que con DSDV y ninguna con DSR.

Tiempo de establecimiento de la comunicación: como se puede observar en las Fig. 3 a 8, el establecimiento de la comunicación es más rápido en el protocolo AODV. Incluso hay casos en los que mediante el uso de protocolos como DSR y DSDV no se logra la comunicación durante el tiempo de simulación. En la Fig. 5 se observa que en el nodo 6, usando el protocolo AODV, se establece comunicación después de 2 segundos, pero se interrumpe, por el movimiento de los nodos. Pero más adelante se restablece. En la Fig. 6 se puede ver que con el uso del protocolo AODV en el nodo 63, cerca a los 4 segundos se logra establecer la comunicación pero, dura muy poco tiempo. De la Fig. 7 se desprende, que con el protocolo DSR, en el nodo 63, se logra establecer la comunicación en varias ocasiones aunque se presenta gran inestabilidad.

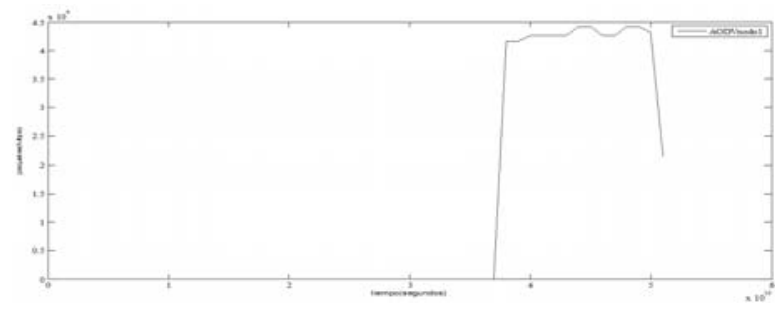

Fig. 3. Troughput en nodo $1 \mathrm{AODV}$. Como se puede ver la comunicación se logra establecer antes de 4 segundos y esta se mantiene estable hasta el final del tiempo de simulación.

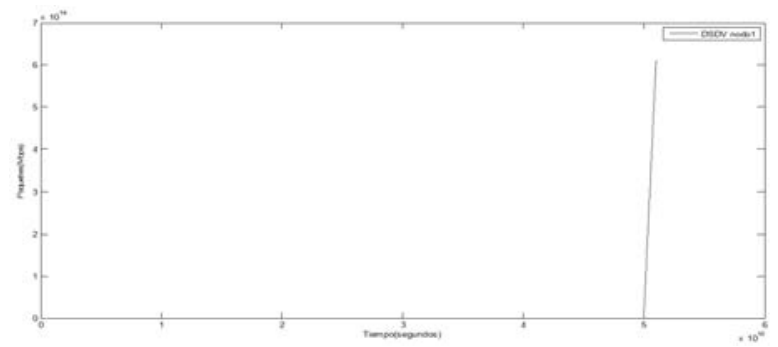

Fig. 4. Troughput en nodo 1DSDV. Se observa que la comunicación no logra esablecerse durante la simulación.

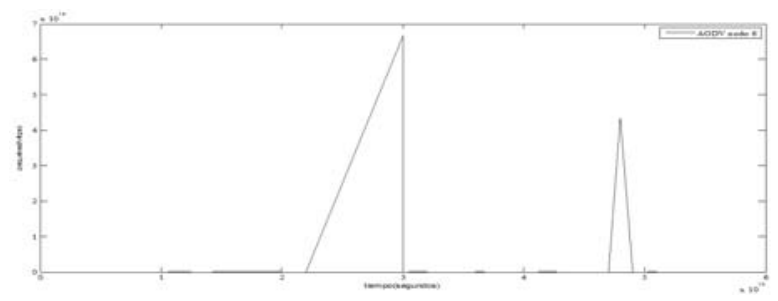

Fig. 5. Troughput en nodo 6 AODV muestra el primer establecimiento de la comunicación, cerca de 2 segundos.

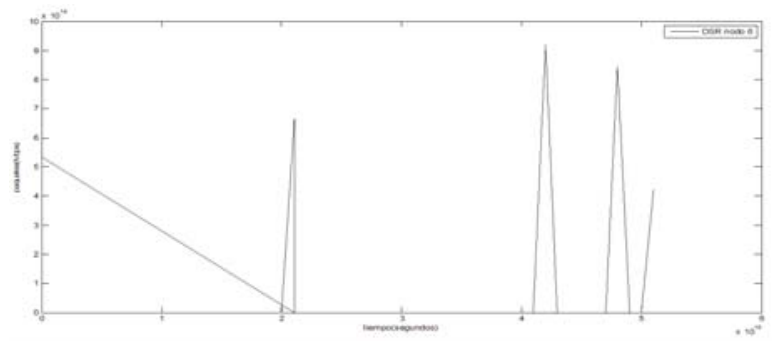

Fig. 6. Troughput en nodo 6 DSR. 


\section{con-ciencias}

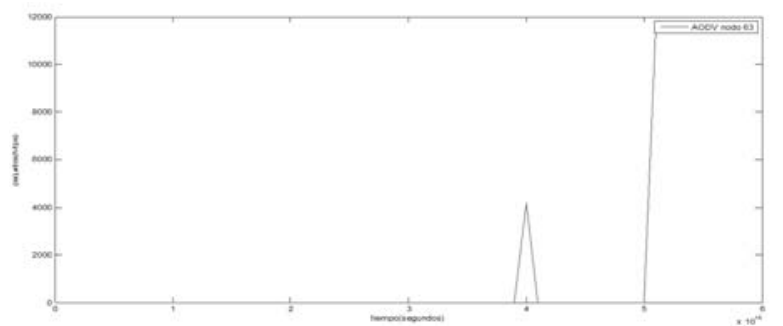

Fig. 7. Troughput en nodo 63AODV.

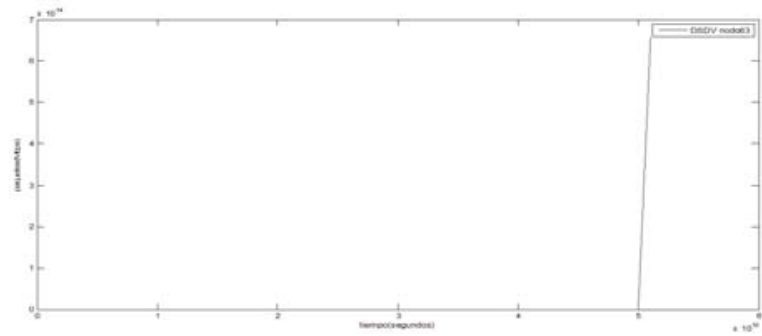

Fig. 8. Troughput en nodo 63DSDV.

Cantidad de paquetes perdidos: en la Fig. 9, se observa que el número de paquetes perdidos aplicando DSR es mayor que utilizando el protocolo AODV, mientras que con DSDV no hay paquetes recibidos ni perdidos ya que no se logra siquiera establecer la comunicacion. Con AODV un porcentaje de 0 perdidas, mientras que con DSR existe un $2,5 \%$.

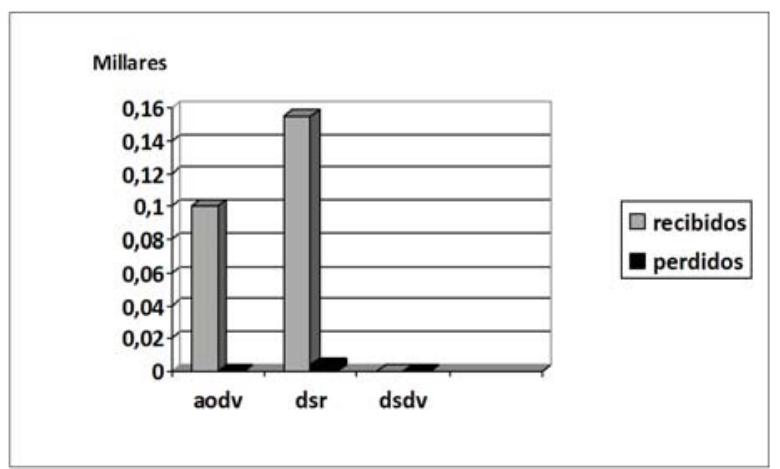

Fig. 9. Comparacion entre paquetes recibidos y paquetes perdidos.

\section{CONCLUSIONES}

Del anterior estudio se desprende que a nivel general el protocolo AODV, presenta un mejor desempeño, ya que con este se logra de manera más rápida el establecimiento de la comunicación, seguido de DSR, mientras que DSDV no estableció comunicación durante el tiempo estimado para la simulación. Resultados que ratifican lo observado en estudios anteriores, que muestran que los protocolos reactivos presentan mejor desempeño, para este tipo de redes, en relación con los proactivos [6], [12], [18], [20] - [22], [24] - [25], [27].

Se puede ver que con el protocolo AODV se reciben menos paquetes que DSR. Esto se debe a que cuando la ruta se inválida, por el movimiento de algún nodo, se envía un mensaje RERR, haciendo que baje la tasa de envió hasta que se descubra una nueva ruta, mientras que en DSR y DSDV, por su funcionamiento no sucede esto. Pero una vez se logra el enlace con AODV, este es más estable. Por otra parte se observa que el porcentaje de paquetes perdidos en AODV es mucho menor, el $0 \%$, mientras que en DSR el porcentaje es mayor $2,5 \%$. Esto es debido a que DSR se demora más en restablecer la ruta, una vez se ha roto el enlace, cuando se presenta mayor congestión en la red. De ahí que se puede decir que en redes pequeñas $\mathrm{y}$ por tanto menos congestionadas, el protocolo DSR puede desempeñarse mejor que AODV, que en vez de ser ventajoso, produciría efectos no deseados como el overead. De esto se desprende que un factor importante al seleccionar el protocolo a utilizar es el tamaño y escalabilidad de la red. De ahí que los estudios sobre los protocolos para las VANETs, pueden dirigirse al planteamiento de protocolos que tengan en cuenta la topología de la red, por ejemplo protocolos como los geocast, que permiten optimizar el mecanismo de descubrimiento, establecimiento y mantenimiento de rutas, al controlar la inundación, teniendo en cuenta la posición geográfica.

\section{REFERENCIAS}


Networks for Inter-vehicle Communications: The Fleenet Project," in symposium on Mobile Ad Hoc Networking and Computing, MobiHOC, New York, 2001.

[2] IEEE. (2005), "802.11", [En línea]. Disponible en: http://standards.ieee.org/ findstds/standard/802.11e-2005.html

[3] T. Kosch, C. Eichler, S. Schroth, C. Strassberger, "The Scalability Problem of Vehicular Ad Hoc Networks and How to Solve it", Wireless Communications IEEE, vol. 13, pp. 22-28, 2006.

[4] C. d. C. d. Bogotá, [En línea]. Disponible en: http://camara.ccb.org.co/documentos/4419_boletin_de_accidentalidad.pdf

[5] P. Torrent, H. Hartenstein, "Fair Sharing of Bandwidth in VANET", in 2nd ACM International Workshop on Vehicular Ad Hoc Networks, 2005.

[6] X. Yang, L. Vaidya, N. Zhao, "A Vehicleto-Vehicle Communication Protocol for Cooperative Collision Warning", in $\mathrm{Mo}$ bile and Ubiquitous Systems: Networking and Services, 2004. MOBIQUITOUS 2004, pp. 113-123, Illinois University, USA, 2004.

[7] I. Calinescy, P.J. Wan, A. Zelikovskya, "Selecting Forwarding Neighbors in Wireless Ad Hoc Networks", in 2nd ACM International Workshop on Vehicular Ad hoc networks, 2005.

[8] The ns-2 Network Simulator, [En línea]. Disponible en: http://www.isi.edu/nsnam/ ns/

[9] IEEE. RFC 3561, [En línea]. Disponible en: http://www.faqs.org/rfcs/rfc3561.html

[10] D. Johnson, Y-C. Hu, DSR: The Dynamic Source Routing Protocol for Multi-Hop Wireless Ad Hoc Networks, [En línea]. Disponible en: http://www.monarch. cs.cmu.edu/

[11] C. E. Perkins, "Highly Dynamic Destination-Sequenced Distance-Vector Routing (DSDV) for Mobile Computers", in SIGCOMM '94 Communications Architectures, Protocols and Applications, 1994.

[12] M. Jaap, Lars Wolf, "Evaluation of Routing Protocols for Vehicular Ad Hoc Networks in City Traffic Scenarios", in 11th EUNICE Open European Summer
School on Networked Applications, Colmenarejo, 2005.

[13] M. Fiore, J. Filali, F. Bonnet, "Vehicular Mobility Simulation for VANETs" Presented at the Simulation Symposium, 2007. ANSS '07. 40th Annual Norfolk, 2007.

[14] IEEE, "802.11p", 2010. [En línea], Disponible en: http://standards.ieee.org/getieee802/download/802.11p-2010.pdf

[15] CIVIS. Cooperative Vehicle Infrastructure Systems, 2010. [En línea] Disponible en: http://www.cvisproject.org/download/ qfree_cvis_brosjyre.pdf

[16] F. Eduardo. Wi-Fi: Nuevos estandares en evolución, 2007. [En línea]. Disponible en: http://www.ceditec.org

[17] S. Elleithy, "Hierarchically Segmented Routing Protocol for MANETs," in American Society for Engineering Education (ASEE 08), New York, USA: United States Military Academy, West Point, 2008.

[18] S. Parma, "Comparative Study and Performance Analysis of FSR, ZRP and AODV Routing Protocols for MANET", in 2nd International Conference and Workshop on Emerging Trends in Technology, 2011.

[19] P. Lakhtaria, N. Patel, Mr. Satish, G. Prajapati, N. Jani, "Securing AODV for MANETs using Message Digest with Secret Key", Network Security \& Its Applications (IJNSA), vol. 1, pp. 111-116, 2009.

[20] Y. Johnson, D. Maltz, D. Maltz, RFC 4728 on The Dynamic Source Routing Protocol (DSR) for Mobile Ad Hoc Networks for IPv4, 2007. [En línea]. Disponible en: http://www.rfc-editor.org/rfc/rfc4728.txt

[21] S. Rakesh, U. Dalal, "A Performance Comparison of Routing Protocols(DSR and TORA) for Security Issue In MANET (Mobile Ad Hoc Networks)", in Mobile Ad-hoc Networks MANETs, 2010.

[22] A. Abd, R. Abdul, "Performance Comparison of AODV, DSDV and I-DSDV Routing Protocols in Mobile Ad Hoc Networks", European Journal of Scientific Research, vol. 31, pp. 566-576, 2009.

[23] N. Young-Bae, "Location-Aided Routing (LAR) in Mobile Ad hoc Networks" Texas AM University, vol. 6, pp. 307-321, 2007. 


\section{con-ciencias}

[24] P. Ferreiro, O. Rubiños, F. Auado, "Analysis of Unicast Routing Protocols for VANETs", presented at the Fifth International Conference on Networking and Services, Valencia, españa, 2009.

[25] J. Bernsen, "Unicast Routing Protocols for Vehicular Ad hoc Networks: A Critical Comparison and Classification", Pervasive and Mobile Computing, pp. 1-18, 2009.

[26] C. d. C. d. Bogota, "Boletin Carrera Sép- tima". Observatorio de Movilidad, 2010. [En línea]. Disponible en:http://camara.ccb.org.co/documentos/7494_boletin_movilidad_Carrera7_edicion_especial_301110.pdf

[27] E. Korkmaz, F. Ozguner and U. Ozguner, Urban MultiHop Broadcast Protocol for InterVehicle Communication Systems, 2004. 